\title{
Guest Editorial Special Issue on Embedded Systems
}

\author{
L. De Micco, Senior Member, IEEE, F. Vargas, Senior Member, IEEE, and P. Fierens, Senior Member, IEEE
}

\section{INTRODUCCIÓN}

\section{$\mathbf{L}$} A temática de sistemas embebidos (SE) tiene una enorme relevancia debido al gran número de aplicaciones en muy diversas áreas. En los últimos años ha habido un interés especial animado por las áreas emergentes de la Internet de las Cosas (IoT, según la sigla en inglés) y la Industria 4.0. Esto puede verse en [1] donde se hace una resumen de la literatura relevante en el área de SE durante los últimos años, repasando brevemente sus componentes y principales aplicaciones tanto en la academia como en la industria.

Como muestra de la relevancia de los SE en Latinoamérica, basta mencionar que para este número especial se han recibido 122 manuscritos. Sólo 33 de esos manuscritos aparecen en este número de IEEE Latin American Transactions. En este artículo introduciremos brevemente cada uno de esos trabajos. Primero, se presenta una breve revisión de la literatura sobre SE [1] en la región de América Latina, y después, se presentan trabajos con aplicaciones en áreas tan diversas como comunicaciones (ver Sección II), satélites (Sección III), transporte (Sección IV), visión por computadora (Sección V), energía (Sección VI) y educación (Sección VII), entre muchos otros ámbitos de aplicación (Sección VIII).

\section{COMUNiCACIONES}

Sin lugar a dudas, la telefonía celular cumple un rol fundamental en la vida diaria. Gutierrez et al. [2] presentan un sistema basado en radio definida por software (SDR, según la sigla en inglés) para realizar mediciones de redes de acceso 4G LTE. Los autores aseguran que su propuesta posee un costo reducido en comparación con otras alternativas basadas en instrumentos especializados.

La cobertura de telefonía celular es deficiente en varias regiones rurales de Latinoamérica. En Gimenez et al. [3] encontramos una propuesta para extender la cobertura de mensajes SMS en zonas rurales utilizando comunicaciones LoRa (Long Range9).

Las redes ad hoc móviles (MANET, según la sigla en inglés) están, en general, basadas sobre sistemas embebidos. Una de las áreas de estudio más relevantes es la de los protocolos de ruteo en MANETs. Silva y colegas [4] presentan un nuevo protocolo de ruteo para redes tolerantes a retrasos (DTN, según

Luciana De Micco is at Instituto de Investigaciones Científicas y Tecnológicas en Electrónica (ICYTE). National University of Mar del Plata and the National Scientific and Technical Research Council (CONICET), Argentina.

Fabian Vargas is at the Catholic University (PUCRS) in Porto Alegre, Brazil.

Pablo I. Fierens is at the Instituto Tecnológico de Buenos Aires (ITBA) and CONICET, Argentina. la sigla en inglés). Basados en simulaciones que toman datos reales de movimientos de buses metropolitanos de la ciudad de Muriaé (Minas Gerais, Brasil), los autores muestran el buen comportamiento del protocolo propuesto aún ante distintas intensidades de tráfico.

La seguridad informática cumple un rol fundamental en todos los sistemas de comunicaciones. Las primitivas criptográficas suelen ser exigentes en cuanto a procesamiento computacional, por lo cual representan un desafío en SEs de bajo poder de cómputo o alimentados por baterías. En el trabajo de Cuiman y colegas [5], encontramos una implementación en procesadores ARM de algoritmos criptográficos basados en emparejamientos bilineales. En particular, los autores presentan una implementación más rápida que otras en la literatura sobre procesadores ARM Cortex-A9.

Los SEs no sólo tienen aplicación en comunicaciones inalámbricas. Bustillos et al. [6] presentan el diseño, implementación y evaluación de una solución embebida para el control de un láser sintonizable con aplicación en multiplexado por longitud de onda (WDM, según la sigla en inglés).

\section{AplicaCiOnES ESPACIALES}

Los diferentes sistemas electrónicos en satélites representan ejemplos prototípicos de sistemas embebidos. En los últimos años ha habido un creciente interés en los nanosatélites o CubeSats debido a su bajo costo comparado con los satélites tradicionales. Ese interés se ve reflejado en la presencia de varios trabajos relacionados con CubeSats en este número especial.

Marcelino et al. [7] describen los sistemas embebidos diseñados para el FloripaSat-1, un CubeSat desarrollado en la Universidade Federal de Santa Catarina (Brasil). Los autores también incluyen resultados experimentales de dos módulos específicos, el sistema de energía o Electrical Power System (EPS) y el sistema responsable de las comunicaciones con las estaciones terrestres o Telemetry, Tracking and Command System (TTC).

Rigo y colegas [8] se concentran en los requerimientos de diseño de circuitos impresos para aplicaciones espaciales, también motivados por el proyecto Floripasat-1. En particular, presentan el diseño de una placa que sigue las reglas de diseño de la Agencia Espacial Europea (ESA, según la sigla en inglés). El diseño no sólo tiene en cuenta reglas para disminuir la interferencia electromagnética, sino que utiliza componentes capaces de resistir amplias variaciones térmicas, funciona en el vacío y tolera la radiación en el espacio.

El trabajo de Rodríguez et al. [9] se enfoca en otro elemento en CubeSats: el receptor GPS. Los autores diseñan un receptor 
con dos antenas que permite procesar las bandas civiles de GPS L1 y L2. Asimismo, presentan mediciones experimentales que validan el correcto funcionamiento del receptor.

\section{TRANSPORTE}

En territorios extensos como los existentes en varios países latinoamericanos, la infraestructura ferroviaria es de vital importancia. Este número incluye tres artículos referidos a aplicaciones de sistemas embebidos en ferrocarriles. Di Vito et al. [10] describen la implementación de un sistema de supervisión de los mecanismos de seguridad de una locomotora. Dorbolo et al. [11] presentan la implementación de un sistema de control automático y monitoreo de barreras en pasos a nivel. Finalmente, el trabajo de Menendez y colegas [12] introduce el diseño e implementación de un sistema de interconexión ferroviaria, basado en FPGAs, que controla automáticamente los cambios de rutas, evitando choques y descarrilamientos.

También encontramos en este número dos artículos referidos a vehículos aéreos no tripulados (UAVs, según la sigla en inglés). Por un lado, de Carvalho Paulino et al. [13] estudian la influencia del ruido en los sensores sobre la navegación autónoma en UAVs. Por otro lado, si bien el foco no son los vehículos no tripulados propiamente dichos, VázquezCarmona y colegas [14] proponen el diseño de un sistema de monitoreo ambiental, basado en Arduino, que es montado sobre un UAV.

\section{VISIÓN POR COMPUTADORA}

La visión por computadora encuentra aplicaciones en muchas áreas, como la seguridad (por ej., la detección de rostros de fugitivos), la medicina (e.g., en el análisis de imágenes médicas) o en la industria (por ej., para la inspección y control de calidad de productos). No es de extrañar, por tanto, que varios de los artículos en la presente edición especial sobre sistemas embebidos estén relacionados con la visión por computadora.

Pérez et al. [15] presentan una arquitectura web modular para el procesamiento de imágenes, compatible con un computador de placa única. Crearon un sistema distribuido de bajo costo y alto rendimiento para el procesamiento de imágenes, integrando placas Raspberry PI, middleware de código abierto, ensamblado web OpenCV.js y un sistema de administración de bases de datos relacionales (RDBMS, según la sigla en inglés) de código abierto como backend.

Muchos sistemas de visión por computadora, además de contar con una parte de procesamiento de imágenes propiamente dicho, se basan en algoritmos de aprendizaje por computadora. Estos algoritmos suelen tener un alto costo computacional que los hace inadecuados para algunas plataformas de hardware de sistemas embebidos. Brandolt Baldissera y Vargas [16] proponen una implementación de redes neuronales convolucionales (CNN, según la sigla en inglés) 2D y 3D, de bajo costo computacional, para la reconocimiento de gestos en tiempo real. Los autores validan su propuesta mediante experimentos con bases de datos disponibles en la literatura, encontrando que obtienen buenos resultados y con mayor velocidad de cómputo que otros algoritmos alternativos.
Abarca et al. [17] proponen una arquitectura de filtrado espacial de imágenes basado en sistemas neuronales tipo P. De acuerdo a sus resultados, los autores afirman que su implementación sobre FPGA es $50 \times 10^{3}$ veces más veloz que las demás soluciones neuromórficas existentes basadas en FPGAs.

El trabajo de Ordoñez, Blotta y Pastore [18] describe la implementación de un algoritmo de seguimiento del movimiento de ojos basado en patrones isocéntricos invariantes. La implementación se realiza sobre una placa de desarrollo que tiene un procesador Cortex M4 y sólo emplea $192 \mathrm{kB}$ de memoria RAM. Los autores evalúan la eficiencia y eficacia de la implementación usando una base de imágenes propia, la cual han compartido libremente para permitir a otros investigadores realizar sus propias pruebas.

El último trabajo en el área de procesamiento de imágenes en esta edición especial es el de Leiva et al. [19]. Los autores presentan la implementación en FPGA de un algoritmo de compresión de imágenes sin pèrdida denominado ImageZero. Es interesante notar que, basados en experimentos, los autores encuentran que la velocidad de compresión no se ve fuertemente afectada por el incremento en la resolución de las imágenes.

\section{ENERGÍA}

En este número especial, hay dos trabajos interesantes relacionadas con el área de energía. Por un lado, Alvarez y Sarli [20] presentan una solución al problema de programación óptima de unidades generadoras de electricidad para satisfacer una demanda determinada (conocido como problema de Asignación de Unidades). Los autores proponen una descomposición de un modelo matemático existente en un problema maestro (a resolver en una PC) y un problema esclavo (a resolver en un sistema embebido). De esta forma, Alvarez y Sarli resuelven el inconveniente de las pequeñas empresas de distribución de energía de adquirir herramientas de procesamiento importadas, debido a sus altos costos en dólares.

Por otro lado, el trabajo de Garcia, Souza y Marafão [21] se enfoca en la administración del consumo domiciliario. Estos autores proponen un sistema de medición de consumo eléctrico inteligente que permite inferir el tipo de carga presente (por ej., qué electrodoméstico está siendo usado). Este tipo de sistemas se denomina de monitoreo no intrusivo de cargas (NILM, según la sigla en inglés) y permite una mejor administración del consumo eléctrico por parte de los usuarios.

\section{EDUCACIÓN SOBRE SISTEMAS EMEBEBIDOS}

Siendo de tanto interés el área de los sistemas embebidos, es importante potenciar plataformas que colaboren con la formación de técnicos y profesionales en la misma. La Computadora Industrial Abierta Argentina (CIAA) es una plataforma para el desarrollo de sistemas embebidos. EDU-CIAA es una versión simplificada, especialmente pensada para el ámbito educativo, que está basada en un microcontrolador LPC4337, con doble núcleo ARM Cortex-M4F y Cortex-M0. Julián, Orosco y Soria [22] presentan el diseño de una placa de extensión 
de la EDU-CIAA para su aplicación en IoT. Dicha placa permite añadirle módulos de comunicación Bluetooth, WiFi y GSM/GPRS, así como varios tipos de sensores. Los autores muestran el desarrollo de un sistema de relevamiento de datos de los sensores desde una aplicación Android.

Una plataforma completa para la educación sobre IoT es presentada por Andrada Tivani et al. [23]. El objetivo es allanar el camino para la experimentación y el aprendizaje de MQTT (MQ Telemetry Transport). La plataforma incluye una simple estación meteorológica, basada en una placa Arduino UNO, que se conecta remotamente con una computadora Raspberry PI 3, donde se encuentra instalado un broker MQTT. El broker usado es Mosquitto y para la programación de flujos MQTT se utiliza Node-RED. Los autores reportan buenos resultados con la utilización de esta plataforma educativa en los primeros cursos donde ha sido usada.

\section{OTRAS APLICACIONES}

Sin lugar a dudas, un área de vital importancia en la región de Latinoamérica es la agricultura. Capraro, Tosetti y Campillo [24] presentan un sistema de riego de precisión, fundamental para regiones de cultivo con pocas precipitaciones. El sistema está compuesto por un conjunto de nodos sensores que se comunican en forma inalámbrica con un nodo central. Éste permite el monitoreo de las condiciones del cultivo y la toma de decisiones. Es interesante notar que los autores han evaluado el funcionamiento del sistema en una plantación de tomate con destino industrial en la provincia de San Juan, Argentina.

Otro tema de gran interés en la región es el de la seguridad de los trabajadores en las explotaciones mineras. Capraro, Segura y Sisterna [25] presentan la implementación de un sistema de localización de trabajadores mineros basado en señales UWB (Ultra-Wide Bandwidth). Estos autores evaluaron su sistema en la mina subterránea Casposo, en la ciudad de Calingasta (San Juan, Argentina).

Los SE también encuentran aplicación en la asistencia a personas con dificultades de movilidad. Villeda Félix et al. [26] desarrollan un sistema, basado comunicaciones de campo cercano con espiras magnéticas, para ayudar a moverse a personas con problemas visuales.

La Industria 4.0 también ocupa un lugar en este número especial. Parsekian et al. [27] presentan una plataforma para el manejo a través de Internet de una máquina con control numérico computarizado (CNC, según la sigla en inglés). El sistema incluye también la detección de fallas durante procesos de manufacturación con la máquina $\mathrm{CNC}$. Los autores del trabajo muestran la implementación y resultados experimentales con máquinas de impresión 3D.

La mejora de la tecnología permite hoy utilizar algoritmos que, hasta hace algunos años, no eran implementables en sistemas embebidos de bajo poder de cómputo. Este es el caso de la reconfiguración parcial dinámica disponible en muchas FPGAs. Esta tecnología permite que diferentes tareas compartan los recursos disponibles en tiempo de ejecución, mediante la descarga de bitstreams parciales en áreas específicas. De allí surge la necesidad de planificar la ubicación de objetos hardware en las zonas de reconfiguración más convenientes según los criterios que se deseen priorizar (consumo de potencia, cantidad de accesos a memoria, etc.), como así también el orden de ejecución de tales reconfiguraciones parciales. En [28] los autores presentan un novedoso sistema de decisión de reconfiguraciones parciales multicriterio. El método propuesto Nuñez Manquez y sus colegas permite la planificación de recursos y de reconfiguraciones en sistemas preemptivos de tiempo real, y es escalable a nuevos criterios de diseño.

Un caso particular de implementación de algoritmos es presentado en el trabajo de Berndsen Peccin et al. [29]. Allí los autores desarrollan una solución para la programación cuadrática con restricciones que tiene aplicación en el control predictivo generalizado (GPC, según la sigla en inglés). El trabajo incluye la validación experimental usando una plataforma FPGA.

Otro caso de implementación de nuevos algoritmos en sistemas embebidos es el sistema desarrollado por De Micco et al. [30]. Allí los autores implementan en FPGA un algoritmo de ordenamiento híbrido que ejecuta instrucciones secuenciales y concurrentes, obteniendo una complejidad menor que algoritmos de ordenamiento conocidos. También reportan una reducción en consumo de recursos, retardo y latencia.

Para terminar de comprender la gran variedad de aplicaciones de los SE, tal vez los siguientes tres trabajos sean suficientes. Conde y colegas [31] presentan un sistema embebido que mejora el proceso de deposición de materiales por ablación con láser pulsado. Su diseño incluye actuadores robustos manejados por motores paso-a-paso y controlados por una placa Arduino. Aguilar-Avelar et al. [32] desarrollan un SE para el control de flujo y presión de un dispositivo para aplicaciones microfluídicas. El sistema, basado en un microcontrolador PIC18F45K50, incluye el control de una bomba de diafragma, una válvula proporcional, un sensor de presión y otro de flujo. Cabrerizo-Morales et al. [33] diseñaron un sistema para el monitoreo de estructuras flotantes. Los autores presentan resultados experimentales sobre un modelo a escala (1:22) del dique seco de Campamento, en Algeciras (España).

\section{AGRADECIMIENTOS}

Deseamos agradecer especialmente el aporte y la discusión con los miembros del comité editorial invitado de este número especial sobre sistemas embebidos.

\section{REFERENCIAS}

[1] L. De Micco, F. L. Vargas, and P. I. Fierens, "A literature review on embedded systems," In this issue., 2020.

[2] D. Gutierrez, F. Gimenez, C. Zerbini, and G. Riva, "Measurement of 4g lte cells with sdr technology," In this issue., 2020.

[3] F. Gimenez, C. Zerbini, and G. Riva, "Extending sms service coverage in rural areas by using lora communication technology," In this issue., 2020.

[4] R. F. Silva, P. H. A. Braga, H. d. A. Mendes, and M. O. Aguiar, "Adaptive: An adaptive routing protocol for vehicle delay-tolerant networks," In this issue., 2020. 
[5] R. Cuiman Márquez, A. J. Cabrera Sarmiento, and S. Sánchez-Solano, "Implementing cryptographic pairings on ARM dual-core processors," In this issue., 2020.

[6] M. C. Bustillos Barcaya and G. F. Rinalde, "Embedded tunable laser control for wdm optical communications systems," In this issue., 2020.

[7] G. M. Marcelino, S. V. Martinez, L. O. Seman, L. K. Slongo, and E. A. Bezerra, "A critical embedded system challenge: The floripasat-1 mission," In this issue., 2020.

[8] C. A. Rigo, L. O. Seman, M. D. Berejuck, and E. A. Bezerra, "Printed circuit board design methodology for embedded systems targeting space applications," In this issue., 2020.

[9] S. Rodríguez, J. G. García, G. Scillone, J. G. Díaz, E. M. López, an R. López La Valle, "Dual-antenna dual-band high performance cubesatcompatible gps receiver," In this issue., 2020.

[10] I. M. Di Vito, P. Gomez, and A. Lutenberg, "Supervision system for emergency brake and traction-system isolation on train formations," In this issue., 2020.

[11] L. Dórdolo, S. Germino, G. Ramoscelli, A. Permingeat, C. Mancón, L. Francucci, D. Baliña, A. Laiuppa, and A. Lutenberg, "Modular system for the monitoring and management of railway signaling equipment," In this issue., 2020.

[12] M. Menéndez, F. Larosa, R. Ghignone, N. Alvarez, and A. Lutenberg, "FPGA implementation of a critical railway interlocking system," In this issue., 2020.

[13] Â. de Carvalho Paulino, L. N. F. Guimarães, and E. H. Shiguemori, "Assessment of noise impact on hybrid adaptive computational intelligence multisensor data fusion applied to real-time uav autonomous navigation,' In this issue., 2020.

[14] E. V. Vázquez-Carmona, J. I. Vasquez-Gomez, and J. C. Herrera-Lozada, "Environmental monitoring using embedded systems on uavs," In this issue., 2020.

[15] C. A. Pérez, M. S. Cleva, D. O. Liska, C. R. da Fonseca, and D. C. Aquino, "Low-cost image and video processing using high-performance middleware in single-board computers with open internet standards," In this issue., 2020.

[16] F. Brandolt Baldissera and F. Vargas, "A light implementation of a 3d convolutional network for online gesture recognition," In this issue., 2020.

[17] M. Abarca, G. Sánchez, J. G. García, L. Avalos, T. Frías, K. Toscano, and H. Pérez, "A scalable neuromorphic architecture to efficiently compute spatial image filtering of high image resolution and size," In this issue., 2020.

[18] C. E. Ordoñez, E. L. Blotta, and J. I. Pastore, "Isophote-based lowcomputing-power eye-tracking embedded-system," In this issue., 2020.

[19] L. Leiva, M. Vázquez, M. Tosini, O. Goñi, and J. Noguera, "FPGA based implementation of imagezero compression algorithm," In this issue., 2020.

[20] G. E. Alvarez and J. L. Sarli, "Optimization of electricity distribution in latin america by using embedded systems," In this issue., 2020.

[21] F. D. Garcia, W. A. Souza, and F. P. Marafão, "Embedded NILM as home energy management system: A heterogeneous computing approach," In this issue., 2020.

[22] J. M. Julián, E. C. Orosco, and C. Soria, "Multi-sensor embedded system with multiple communications based on EDU-CIAA," In this issue., 2020.

[23] A. E. Andrada Tivani, R. M. Murdocca, C. F. Sosa Páez, and J. D. Dondo Gazzano, "Didactic prototype for teaching the mqtt protocol based on free hardware boards and node-red," In this issue., 2020.

[24] F. Capraro, S. Tosetti, and P. Campillo, "Sensor network for monitoring and fault detection in drip irrigation systems based on embedded systems," In this issue., 2020.

[25] F. Capraro, M. Segura, and C. Sisterna, "Human real time localization system in underground mines using uwb," In this issue., 2020.

[26] B. Villeda Félix, J. H. Arroyo Núñez, and R. S. y Núñez Cruz, "Displacement aid system for people with visual impairment using magnetic loops," In this issue., 2020

[27] P. H. L. Parsekian, F. Y. Watanabe, D. A. L. V. Cunha, M. C. Branciforti, and R. V. Aroca, "Failure monitoring and recovery system during manufacturing process," In this issue., 2020.

[28] A. Nuñez Manquez, J. Dondo Gazzano, M. Berón, C. Sosa Paez, an M. Murdocca, "Scheduling and resource planner based on multicriteria method for partially dynamically reconfigurable systems," In this issue. 2020.

[29] V. Berndsen Peccin, D. Martins Lima, and J. E. Costa Flesch, Rodolfo Césarand Normey-Rico, "Fast constrained generalized predictive control with admm embedded in an fpga," In this issue., 2020.
[30] L. De Micco, M. L. Acosta, and M. Antonelli, "Hybrid sorting algorithm implemented by high level synthesis," In this issue., 2020.

[31] J. M. Conde Garrido, C. Kuo, and J. M. Silveyra, "Control system for the synthesis of thin films by pulsed laser deposition," In this issue., 2020.

[32] C. Aguilar-Avelar, M. Esparza, F. Chacón, D. Aráiz-Hernández, B. SotoGarcía, J. F. Y. de León, L. P. Velarde-Calvillo, and A. Abarca-Blanco, "Fully embedded flow control device for microfluidic applications," In this issue., 2020.

[33] M. A. Cabrerizo-Morales, C. Rodriguez-Sanchez, J. Vaquero, A. Rodriguez-Moreno, and S. Borromeo, "New methodology and instrumental system for floating structures monitoring and analysis," In this issue., 2020. 


\title{
Guest Editorial Special Issue on Embedded Systems
}

\author{
L. De Micco, Senior Member, IEEE, F. Vargas, Senior Member, IEEE, and P. Fierens, Senior Member, IEEE
}

\section{INTRODUÇÃO}

$\mathbf{O}$ Tema de sistemas embarcados (SE) tem uma enorme relevância devido ao grande número de aplicações em áreas muito diversas, nos últimos anos tem havido um interesse especial impulsionado pelas áreas emergentes da Internet das Coisas (IoT, de acordo com a sigla em inglês) e Industry 4.0. Isto pode ser visto em [1], onde é feito um resumo da literatura relevante na área de SE durante o últimos anos, revisando brevemente seus componentes e principais aplicações tanto na academia quanto na indústria.

Como sinal da relevância de SE na América Latina, basta mencionar que, para esse número especial, foram recebidos 122 manuscritos. Destes, apenas 33 aparecem nesta edição de IEEE Latin American Transactions. Neste artigo introduziremos brevemente cada um desses trabalhos. Primeiro, é apresentada uma breve revisão da literatura sobre SE [1] na região da América Latina e, em seguida, trabalha com aplicações em áreas tão diversas quanto comunicações (Seção II), satélites (Seção III), transporte (Seção IV), visão computacional (Seção V), energia (Seção VI) e educação (Seção VII), entre muitas outras áreas de aplicação (Seção VIII).

\section{COMUNiCAÇÕES}

Sem dúvida, o celular desempenha um papel fundamental na vida cotidiana. Gutierrez et al. [2] apresentam um sistema de rádio baseado em software (SDR, de acordo com sigla em inglês) para realizar medições de redes de acesso 4G LTE. Os autores asseguram que sua proposta tenha um custo reduzido em comparação com outras alternativas em instrumentos especializados. A cobertura do telefone celular é deficiente em várias regiões rurais da América Latina. Em Gimenez et al. [3] encontramos uma proposta para estender a cobertura de mensagens SMS em áreas rurais usando comunicações LoRa (Long Range9).

Redes ad hoc para celular (MANET, de acordo com a sigla em inglês) são, em geral, baseadas em sistemas embarcados. Uma das áreas de estudo mais relevantes são as dos protocolos de roteamento em MANETs. Silva e colegas [4] apresentam um novo protocolo de roteamento para redes tolerantes a atraso (DTN, de acordo com o acrônimo em inglês). Com base em simulações que recebem dados de movimentos reais de ônibus metropolitanos da cidade de Muriaé (Minas Gerais, Brasil), os autores mostram o bom comportamento do protocolo proposto em relação a diferentes intensidades de tráfego. A segurança desempenha um papel fundamental em sistemas de comunicação. As primitivas criptográficas geralmente são muito exigentes em termos de processamento computacional, e portanto, representam um desafio em SE de baixo poder de computação e/ou bateria. No trabalho de Cuiman e colegas [5], encontramos uma implementação em processadores ARM de algoritmos criptográficos baseados em emparelhamentos bilineares. Em particular, os autores apresentam uma implementação mais rápida do que outros na literatura envolvendo processadores ARM Cortex-A9. Os SE não têm apenas aplicação em comunicações sem fio. Bustillos et al. [6] apresentam o projeto, implementação e avaliação de uma solução embarcada para o controle de um laser sintonizável com aplicação em multiplexação por comprimento de onda (WDM, de acordo com a sigla em inglês).

\section{APLicaç̃̃es ESPaCiais}

Os diferentes sistemas eletrônicos nos satélites representam exemplos típicos de sistemas embarcados. Nos últimos anos, tem havido um interesse crescente em nanossatélites ou CubeSats devido ao seu baixo custo comparado aos satélites tradicionais. Esse interesse se reflete na presença de vários trabalhos relacionados a CubeSats nesta edição especial. Marcelino et al. [7] descrevem sistemas embarcados projetados para o FloripaSat-1, um CubeSat desenvolvido na Universidade Federal de Santa Catarina (Brasil). Os autores também incluem resultados experimentais de dois módulos específicos, o sistema de energia ou Electrical Power System (EPS) e o sistema responsável pelas comunicações com estações terrestres, o Telemetry, Tracking and Command System (TTC).

Rigo e colegas [8] se concentram nos requisitos de projeto de circuitos impressos para aplicações espaciais, também motivados pelo projeto Floripasat-1. Em particular, eles apresentam o desenho de uma placa que segue as regras de projeto da Agência Espacial Europeia (ESA, de acordo com a sigla em Inglês) $O$ design não apenas leva em consideração as regras para diminuir a interferência eletromagnética, mas usa componentes capazes de suportar grandes variações térmicas, trabalha no vácuo e tolera a radiação espaçial. O trabalho de Rodríguez et al. [9] concentra-se em outro elemento do CubeSats: o receptor GPS. Os autores projetam um receptor com duas antenas que permitem processar as bandas civis de GPS L1 e L2. Apresentam também medidas experimentais que validam o correto funcionamento do receptor.

\section{TRANSPORTE}

Em grandes territórios como os de vários países Latinoamericanos, a infraestrutura ferroviária é de vital importância Esse número inclui três artigos referentes a aplicações de 
sistemas embarcados em ferrovias. Say Vito et al. [10]descrevem a implementação de um sistema de monitoramento dos mecanismos de segurança de uma locomotiva. Dorbolo et al. [11] apresentam a implementação de um sistema controle automático e monitoramento de barreiras nas passagens de nível. Finalmente, o trabalho de Menendez e colegas [12] introduz a concepção e implementação de um sistema de interligação ferroviária, baseada em FPGAs, que controla automaticamente mudanças de rota, evitando falhas e descarrilamentos. Também encontramos nesta edição dois artigos envolvendo veículos aéreos não tripulados (UAVs, de acordo com a sigla em Inglês). Por um lado, por Carvalho Paulino et al. [13] estudam a influência do ruído em sensores para navegação autônoma em UAVs. Por outro lado, embora o foco não seja os próprios veículos não tripulados, VázquezCarmona e colegas [14] propõem o projeto de um sistema de monitoramento ambiental, baseado em Arduino, montado sobre um UAV.

\section{VISÃO POR COMPUTADOR}

A visão computacional encontra aplicações em muitas áreas, como segurança (por exemplo, na detecção de rostos de fugitivos), medicina (na análise de imagens médicas) ou na indústria (para inspeção e controle de qualidade de produto). Não é de admirar, portanto, que vários dos artigos desta edição especial sobre sistemas embarcados estão relacionados à visão computacional. Pérez et al. [15] apresentam uma arquitetura web modular para processamento de imagem compatível com um computador de praca única Eles criaram um sistema distribuído de baixo custo e alto desempenho para o processamento de imagens, integrando placas Raspberry PI, middleware de código aberto, Web assembly OpenCV.js e um sistema de administração de bases de dados relacionais (RDBMS, de acordo com a sigla em inglês) de código aberto como backend. Muitos sistemas de visão computacional, além de contar com uma parte de processamento de imagens, propriamente dito, também são baseados em algoritmos de aprendizado computacional. Esses algoritmos geralmente têm um alto custo computacional, o que os torna inadequados para algumas plataformas de hardware de sistemas embarcados. Brandolt Baldissera e Vargas [16] propõem uma implementação de redes neurais convolucionais ( $\mathrm{CNN}$, na sigla em inglês) 2D e $3 \mathrm{D}$, de baixo custo computacional, para reconhecimento de gestos em tempo real. Os autores validam sua proposta através de experiências com banco de dados disponíveis na literatura, indicando que obtêm bons níveis de reconhecimento com maior velocidade de computação que outros algoritmos alternativos. Abarca et al. [17] propõem uma arquitetura de filtragem espacial de imagens baseada em sistemas neurais do tipo P. De acordo com seus resultados, os autores afirmam que sua implementação sobre FPGA é $50 \times 10^{3}$ vezes mais rápida do que outras soluções neuromórficas existentes baseadas em FPGAs. O trabalho de Ordoñez, Blotta e Pastore [18] descreve a implementação de um algoritmo de rastreamento de movimento de olhos baseados em padrões isocêntricos invariantes. Implementação é realizada em uma placa de desenvolvimento que possui um processador Cortex M4 e usa apenas $192 \mathrm{kB}$ de memória RAM. Os autores avaliam a eficiência e a eficácia da implementação usando sua própria base de imagens, que compartilharam livremente para permitir que outros pesquisadores façam seus próprios testes. O último trabalho na área de processamento de imagens desta edição especial é a de Leiva et al. [19].Os autores apresentam a implementação em FPGA de um algoritmo de compressão de imagens sem perdas chamada ImageZero. É interessante notar que, com base em experimentos, os autores eles sugerem que a velocidade de compressão não é sensivelmente afetada pelo aumento da resolução das imagens.

\section{ENERGIA}

Nesta edição especial, existem dois trabalhos interessantes relacionados à área de energia. Por um lado, Alvarez e Sarli [20] apresentam uma solução para o problema de programação ótima de unidades geradoras de eletricidade para atender a uma demanda específica (conhecida como Problema de Atribuição de Unidades). Os autores propõem a decomposição de um modelo matemático existente em um problema principal (a ser resolvido em um PC) e um problema escravo (a ser resolvido em um sistema embarcado). Desta forma, Alvarez e Sarli resolvem a dificuldade que pequenas empresas de distribuição de energia enfrentam para adquirir ferramentas importadas para o processamento de dados, devido ao alto custo envolvido, em dólar. Por outro lado, o trabalho de Garcia, Souza e Marafão [21] concentra-se na gestão do consumo doméstico. Estes autores propõem um sistema de medição de consumo elétrico inteligente que permite inferir o tipo de carga presente (por exemplo, qual aparelho está sendo usado). Este tipo de sistema é denominado de monitoramento de carga não intrusiva (NILM, de acordo com a sigla em inglês) e permite uma melhor administração do consumo de eletricidade por parte dos usuários.

\section{EDUCAÇÃO EM SISTEMAS EMBARCADOS}

A área de sistemas embarcados sendo de tal interesse, é importante potencializar plataformas que colaborem com o treinamento de técnicos e profissionais na mesma. O Computador Industrial Aberto Argentino (CIAA) é uma plataforma para o desenvolvimento de sistemas embarcados. EDU-CIAA é uma versão simplificada, especialmente projetado para a educação, e é baseado em um microcontrolador LPC4337 com duplo ARM Cortex-M4F e um núcleo Cortex-M0. Julian, Orosco e Soria [22] apresentam o projeto de uma placa de extensão do EDU-CIAA para aplicação em IoT. Esta placa permite adicionar módulos de comunicação Bluetooth, WiFi e GSM / GPRS, bem como vários tipos de sensores. Os autores apresentam o desenvolvimento de um sistema de pesquisa de dados de sensores a partir de um aplicativo Android. Uma plataforma completa para a educação da IoT é apresentadA por Andrada Tivani et al. [23].O objetivo é pavimentar o caminho para a experimentação e aprendizagem de MQTT (MQ Telemetry Transport). A plataforma inclui uma estação meteorológica simples, baseada em uma placa Arduino UNO, que se conecta remotamente a um computador Raspberry PI 3 , onde se encontra instalado um broker MQTT. O broker 
utilizado é Mosquitto, e para a programação dos fluxos MQTT se utiliza o Node-RED. Os autores relatam bons resultados com o uso desta plataforma educacional nos primeiros cursos em que foi empregada.

\section{OUTRAS APLICAÇÕES}

Sem dúvida, uma área de vital importância na América Latina é a agricultura. Capraro, Tosetti e Campillo [24] apresentam um sistema de irrigação de precisão, essencial para regiões de crescimento com pouca chuva. O sistema é composto por um conjunto de nós sensores que se comunicam sem fio com um nó central. Isso permite o monitoramento das condições da colheita e tomada de decisão. É interessante notar que os autores apresentam resultados de avaliação da operação do sistema em uma plantação de tomate com destino industrial, na província de San Juan, Argentina. Outro tópico de grande interesse na região é o de segurança de trabalhadores em operações de mineração. Capraro, Segura e Sisterna [25] apresentam a implementação de um sistema de rastreamento de trabalhadores de mineração com base em Sinais UWB (UltraWide Bandwidth). Esses autores avaliaram seu sistema na mina subterrânea de Casposo, na cidade de Calingasta (San Juan, Argentina). SE também encontram aplicação em assistência para pessoas com dificuldades de mobilidade. Villeda Felix et al. [26] desenvolveram um sistema baseado em comunicações de campo próximo com curvas magnéticas, para ajudar pessoas com problemas visuais a moverem-se. A indústria 4.0 também ocupa um lugar nesta edição especial. Parsekian et al. [27] apresentam uma plataforma para operação de uma máquina com controle numérico computadorizado (CNC, de acordo com a sigla em inglês) via Internet. O sistema também inclui detecção de falhas na máquina CNC durante o processo de fabricação. Os autores do trabalho mostram a implementação e os resultados experimentais com máquinas de impressão 3D. Atualmente, o avanço da tecnologia permite o uso de algoritmos que, até alguns anos atrás, não eram implementáveis em sistemas embarcados com baixo poder computacional. Este é o caso da reconfiguração parcial dinâmica, disponível em muitos FPGAs. Esta tecnologia permite, a diferentes tarefas, compartilhar recursos disponíveis em tempo de execução, mediante o download parcial de bits de configuração, em áreas específicas do componente. A partir daí, surge a necessidade de planejar a localização de objetos de hardware em zonas de reconfiguração mais adequadas, de acordo com os critérios a serem priorizados (consumo de potência, número de acessos à memória, etc.), como também a ordem de execução de tais reconfigurações parciais. Em [28] os autores apresentam um novo sistema de decisão de reconfiguração parcial baseado em vários critérios. Neste método proposto, Nuñez Manquez e seus colegas permitem o planejamento de recursos e de reconfiguração do sistema preemptivo em tempo real, e é escalável para novos critérios de projeto. Um caso particular de implementação de algoritmos é apresentado no trabalho de Berndsen Peccin et al. [29]. Lá, os autores desenvolvem uma solução para programação quadrática com restrições que tem aplicação no controle preditivo generalizado (GPC, de acordo com a sigla em inglês). $O$ trabalho inclui validação experimental usando uma plataforma FPGA. Outro caso de implementação de novos algoritmos em sistemas embarcados é o sistema desenvolvido por De Micco et al. [30]. Lá, os autores implementam um algoritmo de ordenação híbrido no FPGA que executa instruções sequenciais e simultâneas, produzindo assim uma complexidade computacional menor do que os algoritmos de classificação conhecidos. Eles também relatam uma redução no consumo de recursos, atraso e latência. Para concluir a compreensão da grande variedade de aplicações de SE, talvez os três trabalhos a seguir sejam suficientes. Count e colegas [31] apresentam um sistema embarcado que melhora o processo de deposição de materiais por ablação a laser pulsado. Sua técnica inclui atuadores robustos acionados por motores de passo e controlados por uma placa Arduino. Aguilar-Avelar et al. [32] desenvolveram um SE para o controle de fluxo e pressão de um dispositivo para aplicações microfluídicas. O sistema, baseado em um microcontrolador PIC18F45K50, inclui o controle de uma bomba de diafragma, uma válvula proporcional, um sensor de pressão e um sensor de fluxo. Cabrerizo-Morales et al. [33] projetaram um sistema para monitorar estruturas flutuantes. Os autores apresentam resultados experimentais em uma maquete (escala 1:22) da doca seca de Campamento, em Algeciras (Espanha).

\section{AgRAdecimentos}

Desejamos agradecer especialmente a contribuição e discussão com os membros do comitê editorial convidado desta edição especial em sistemas embarcados.

\section{REFERENCIAS}

[1] L. De Micco, F. L. Vargas, and P. I. Fierens, "A literature review on embedded systems," In this issue., 2020.

[2] D. Gutierrez, F. Gimenez, C. Zerbini, and G. Riva, "Measurement of 4g lte cells with sdr technology," In this issue., 2020.

[3] F. Gimenez, C. Zerbini, and G. Riva, "Extending sms service coverage in rural areas by using lora communication technology," In this issue., 2020.

[4] R. F. Silva, P. H. A. Braga, H. d. A. Mendes, and M. O. Aguiar, "Adaptive: An adaptive routing protocol for vehicle delay-tolerant networks," In this issue., 2020.

[5] R. Cuiman Márquez, A. J. Cabrera Sarmiento, and S. Sánchez-Solano, "Implementing cryptographic pairings on ARM dual-core processors," In this issue., 2020.

[6] M. C. Bustillos Barcaya and G. F. Rinalde, "Embedded tunable laser control for wdm optical communications systems," In this issue., 2020.

[7] G. M. Marcelino, S. V. Martinez, L. O. Seman, L. K. Slongo, and E. A. Bezerra, "A critical embedded system challenge: The floripasat-1 mission," In this issue., 2020.

[8] C. A. Rigo, L. O. Seman, M. D. Berejuck, and E. A. Bezerra, "Printed circuit board design methodology for embedded systems targeting space applications," In this issue., 2020.

[9] S. Rodríguez, J. G. García, G. Scillone, J. G. Díaz, E. M. López, and R. López La Valle, "Dual-antenna dual-band high performance cubesatcompatible gps receiver," In this issue., 2020.

[10] I. M. Di Vito, P. Gomez, and A. Lutenberg, "Supervision system for emergency brake and traction-system isolation on train formations," In this issue., 2020.

[11] L. Dórdolo, S. Germino, G. Ramoscelli, A. Permingeat, C. Mancón, L. Francucci, D. Baliña, A. Laiuppa, and A. Lutenberg, "Modular system for the monitoring and management of railway signaling equipment," In this issue., 2020.

[12] M. Menéndez, F. Larosa, R. Ghignone, N. Alvarez, and A. Lutenberg, "FPGA implementation of a critical railway interlocking system," In this issue., 2020 . 
[13] Â. de Carvalho Paulino, L. N. F. Guimarães, and E. H. Shiguemori, "Assessment of noise impact on hybrid adaptive computational intelligence multisensor data fusion applied to real-time uav autonomous navigation," In this issue., 2020.

[14] E. V. Vázquez-Carmona, J. I. Vasquez-Gomez, and J. C. Herrera-Lozada, "Environmental monitoring using embedded systems on uavs," In this issue., 2020.

[15] C. A. Pérez, M. S. Cleva, D. O. Liska, C. R. da Fonseca, and D. C. Aquino, "Low-cost image and video processing using high-performance middleware in single-board computers with open internet standards," In this issue., 2020.

[16] F. Brandolt Baldissera and F. Vargas, "A light implementation of a 3d convolutional network for online gesture recognition," In this issue., 2020.

[17] M. Abarca, G. Sánchez, J. G. García, L. Avalos, T. Frías, K. Toscano, and H. Pérez, "A scalable neuromorphic architecture to efficiently compute spatial image filtering of high image resolution and size," In this issue., 2020.

[18] C. E. Ordoñez, E. L. Blotta, and J. I. Pastore, "Isophote-based lowcomputing-power eye-tracking embedded-system," In this issue., 2020.

[19] L. Leiva, M. Vázquez, M. Tosini, O. Goñi, and J. Noguera, "FPGA based implementation of imagezero compression algorithm," In this issue., 2020.

[20] G. E. Alvarez and J. L. Sarli, "Optimization of electricity distribution in latin america by using embedded systems," In this issue., 2020.

[21] F. D. Garcia, W. A. Souza, and F. P. Marafão, "Embedded NILM as home energy management system: A heterogeneous computing approach," In this issue., 2020.

[22] J. M. Julián, E. C. Orosco, and C. Soria, "Multi-sensor embedded system with multiple communications based on EDU-CIAA," In this issue. 2020.

[23] A. E. Andrada Tivani, R. M. Murdocca, C. F. Sosa Páez, and J. D. Dondo Gazzano, "Didactic prototype for teaching the mqtt protocol based on free hardware boards and node-red," In this issue., 2020.

[24] F. Capraro, S. Tosetti, and P. Campillo, "Sensor network for monitoring and fault detection in drip irrigation systems based on embedded systems," In this issue., 2020.

[25] F. Capraro, M. Segura, and C. Sisterna, "Human real time localization system in underground mines using uwb," In this issue., 2020.

[26] B. Villeda Félix, J. H. Arroyo Núñez, and R. S. y Núñez Cruz, "Displacement aid system for people with visual impairment using magnetic loops," In this issue., 2020

[27] P. H. L. Parsekian, F. Y. Watanabe, D. A. L. V. Cunha, M. C. Branciforti, and R. V. Aroca, "Failure monitoring and recovery system during manufacturing process," In this issue., 2020.

[28] A. Nuñez Manquez, J. Dondo Gazzano, M. Berón, C. Sosa Paez, an M. Murdocca, "Scheduling and resource planner based on multicriteria method for partially dynamically reconfigurable systems," In this issue. 2020.

[29] V. Berndsen Peccin, D. Martins Lima, and J. E. Costa Flesch, Rodolfo Césarand Normey-Rico, "Fast constrained generalized predictive control with admm embedded in an fpga," In this issue., 2020.

[30] L. De Micco, M. L. Acosta, and M. Antonelli, "Hybrid sorting algorithm implemented by high level synthesis," In this issue., 2020.

[31] J. M. Conde Garrido, C. Kuo, and J. M. Silveyra, "Control system for the synthesis of thin films by pulsed laser deposition," In this issue., 2020.

[32] C. Aguilar-Avelar, M. Esparza, F. Chacón, D. Aráiz-Hernández, B. SotoGarcía, J. F. Y. de León, L. P. Velarde-Calvillo, and A. Abarca-Blanco, "Fully embedded flow control device for microfluidic applications," In this issue., 2020

[33] M. A. Cabrerizo-Morales, . C. Rodriguez-Sanchez, J. Vaquero, A. Rodriguez-Moreno, and S. Borromeo, "New methodology and instrumental system for floating structures monitoring and analysis," In this issue., 2020

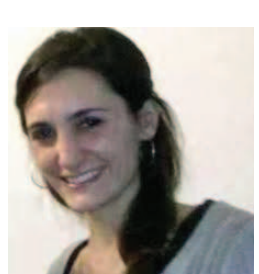

Luciana De Micco Electronics Engineer (2004) and Engineering Doctor with Mention in Electronics (2009), Facultad de Ingeniería, Universidad Nacional de Mar Del Plata - UNMDP, Argentina. De Micco is director of the Laboratory of Chaotic Systems at the Instituto de Investigaciones Científicas y Tecnológicas en Electrónica (ICYTE). She is Adjunct Researcher at the National Scientific and Technical Research Council (CONICET), Argentina. She is Category 3 Research Professor, according to the Comisión Nacional de Categorización, Ministry of Education, Argentina. Luciana is also a Junior Associate Researcher at the Abdus Salam International Centre for Theoretical Physics (ICTP). She is Adjunct Professor at the Universidad Nacional de Mar del Plata. De Micco is interested in chaotic systems, its digitalization and implementation in digital devices.

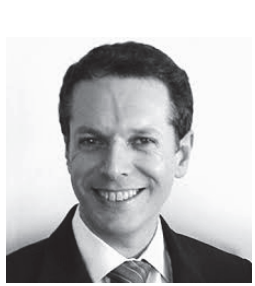

Fabian Luis Vargas Fabian Vargas obtained his Ph.D. Degree in Microelectronics from the Institut National Polytechnique de Grenoble (INPG), France, in 1995. At present, he is Full Professor at the Catholic University (PUCRS) in Porto Alegre, Brazil. He works in the area of computer systems architecture focusing on robustness and security for critical applications. Prof. Vargas is "Level-1" researcher of the Brazilian National Science Foundation $(\mathrm{CNPq})$ since 1996. He co-founded the IEEEComputer Society Latin American Test Technology Technical Council (LA-TTTC) in 1997 and the IEEE Latin American Test Symposium - LATS (former Latin American Test Workshop - LATW) in 2000. Prof. Vargas has acted as Guest Editor of several journal, such as the Journal of Electronic Testing: Theory and Applications - JETTA (Springer) IEEE Design and Test of Computers Magazine and the Journal of Low-Power Electronics - JOLPE (American Scientific Publishers). He is currently member of the Editorial Board of JETTA. Prof. Vargas received for several times the Meritorious Service Award of the IEEE Computer Society for providing significant services as chair of the IEEE Latin American Regional TTTC Group and the LATS. Prof. Vargas is Senior Member of IEEE and Golden Core Member of the IEEE Computer Society.

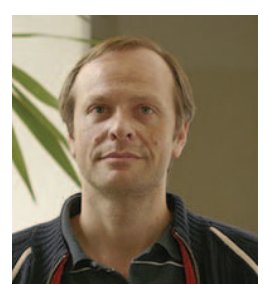

Pablo I. Fierens Electronics Engineer (1997), Instituto Tecnológico de Buenos Aires - ITBA, Argentina. MSc (2000) and PhD (2003), Cornell University, USA Fierens is Full Professor at ITBA. He is director of the Optoelectronics Lab at ITBA. Independent Researcher at CONICET. Associate Editor of IEEE Latin America Transactions. IEEE Senior Member. Pablo is interested in communications, nonlinear optics, and noise in nonlinear systems. 